\title{
Invasive Pulmonary Aspergillosis with Thrombosis in the Left Atrium
}

\author{
Kanako Kobayashi, Shuichi Yano, Shinji Shishido and Takeshi Tokushima
}

\begin{abstract}
We report a female patient with left atrial wall invasion from pulmonary aspergillosis. She had been treated for diabetes mellitus. Pulmonary aspergillosis extended to the left atrial wall via the left pulmonary vein and formed a polypoid lesion in the left atrium. The polypoid lesion was composed of thrombus, and the thrombus increased in size to become large, and it showed invagination into the mitral valve during diastole. We considered that the thrombus was formed on the injured endocardium. Severe invasive aspergillosis thus could occur in a mild systemic immunocompromised host.
\end{abstract}

(Internal Medicine 40: 250-254, 2001)

Key words: invasive pulmonary aspergillosis, left atrium wall invasion

\section{Introduction}

There have been many case reports of invasive pulmonary aspergillosis in immunocompromised hosts. However, there are few reports of cardiac invasion from pulmonary aspergillosis or cardiac fungal infection in the immunocompetent host. In this case, pulmonary aspergillosis invaded the left atrial wall and thrombus was formed on the injured endocardium in the mild systemic immunocompromised host.

\section{Case Report}

On October 28, 1996, a 58-year- old woman was transferred to our hospital with a low grade fever and an increase of CRP and abnormal lung infiltrative shadow on the chest radiograph. Although she had been treated at another hospital with ceftazidime, imipenem, and minocycline hydrochloride, her condition had not improved. She had been treated for diabetes mellitus with insulin since 1993. However, the treatment of diabetes mellitus was not enough, as the glycosylated hemo- globin $\mathrm{A}_{1 \mathrm{c}}$ was $7.8 \%$ on admission. There was no history of cardiovascular disease, hypertension, or corticosteroid or antibiotic therapy before the present illness.

On admission, her temperature was $37.4^{\circ} \mathrm{C}$, her blood pressure was $120 / 70 \mathrm{mmHg}$, and her pulse rate was 82 beats $/ \mathrm{min}$. Breath sounds were diminished over the left lung. Laboratory tests revealed a white blood cell count of 13,000 with a left shift and a CRP of $7.5 \mathrm{mg} / \mathrm{dl}$. Aspergillus antigen was negative and aspergillus antibody was also negative. The $\gamma$-globulin level was slightly elevated. A chest radiograph showed infiltrative shadow in the left lung (Fig. 1A). Chest CT scans revealed mass lesion with atelectasis of lingula and it extended into the left atrium (LA) (Fig. 1B). The mass in the LA was considered to have invaded from the left lung lesion. Lymphadenopathy in the mediastinum was not found. On bronchofiberscopic examination, the orifices of the left upper and lower lobe bronchi were stenosed by a bloody mass. Transbronchial biopsy of the mass revealed hyphae of aspergillus. Culture of the biopsy material on Sabouraud glucose agar showed white-green colonies. The fungal isolates were stained with lactophenol cotton blue stain and microscopic examination revealed columnar conidial head with semi ball-shaped vesicles and flask-like phialides, findings characteristic of Aspergillus fumigatus. There was no evidence of malignancy in the specimen. Echo-guided transcutaneous needle biopsy of the left lung mass was done, and pathological examination revealed necrotic tissue with fungal hyphae of aspergillus. As she received fungicidal treatment with miconazole for a week without effect, she was given amphotericin B (total dosage $2 \mathrm{~g}$ ) intravenously. However, she had nausea, vomiting and appetite loss. Her laboratory data showed renal dysfunction and hypokalemia. Therefore, we decided to discontinue medication by amphotericin B, and changed to fluconazole. The pulmonary shadow was not improved. A second echo-guided transcutaneous needle lung biopsy also revealed pulmonary aspergillosis. We followed the mass in the LA by transesophageal and transthoracic echocardiography, noting a change in shape and an increase in size. From June 1997, she complained of gradually worsening dyspnea. Chest CT scans showed marked cardiomegaly with bilateral pleural effusion. On July 1, 1997, she suddenly devel-

From the Department of Pulmonary Medicine, National Matsue Hospital, Matsue

Received for publication January 6, 2000; Accepted for publication September 8, 2000

Reprint requests should be addressed to Dr. Kanako Kobayashi, the Department of Pulmonary Medicine, National Matsue Hospital, 5-8-31 Agenogi, Matsue, Shimane 690-8556 
oped left hemiparesis. Brain CT scans revealed no significant abnormalities, and her hemiparesis improved gradually for 45 days. Late in July, her general condition became progressively worse. The systolic blood pressure fell to $80 \mathrm{mmHg}$.
The large LA mass showed diastolic invagination into the mitral valve on echocardiography (Fig. 2). The next day, she suffered cardiopulmonary arrest and died. Postmortem examination revealed a large necrotic mass in the left lung (Fig. 3A).

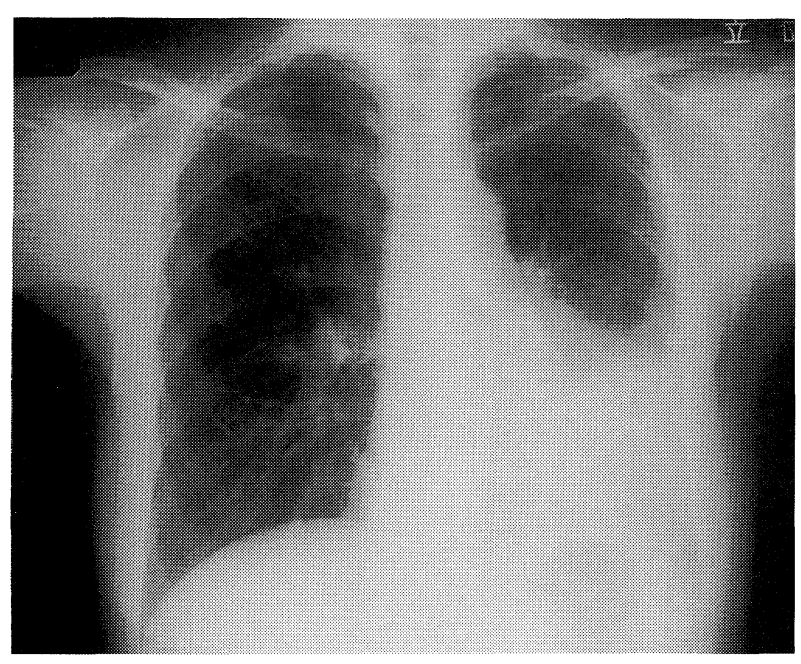

A

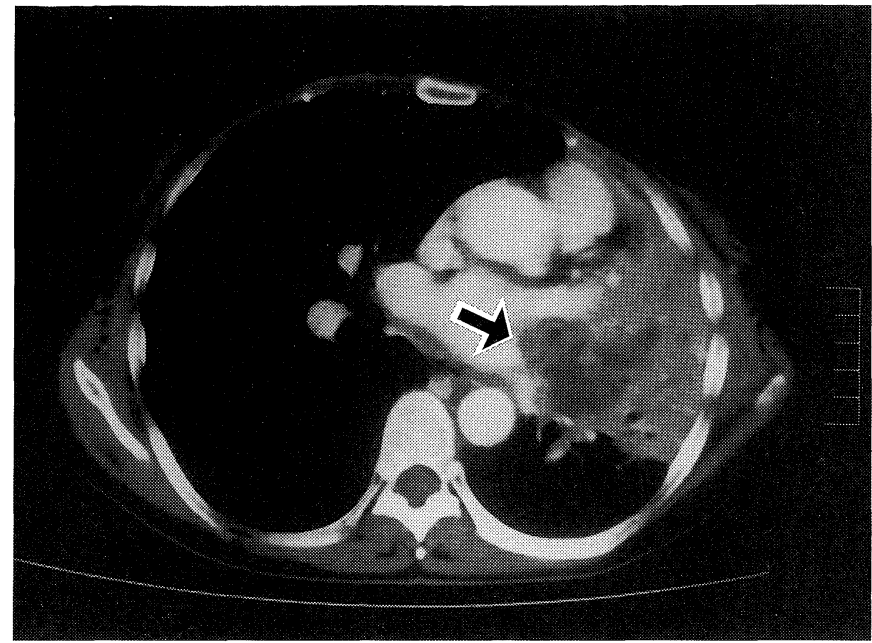

B

Figure 1. A) A chest radiograph obtained on admission demonstrating an infiltrative shadow in the left lung. B) A chest CT scan reveals a mass lesion of left atrium (arrow) and lingula atelectasis.

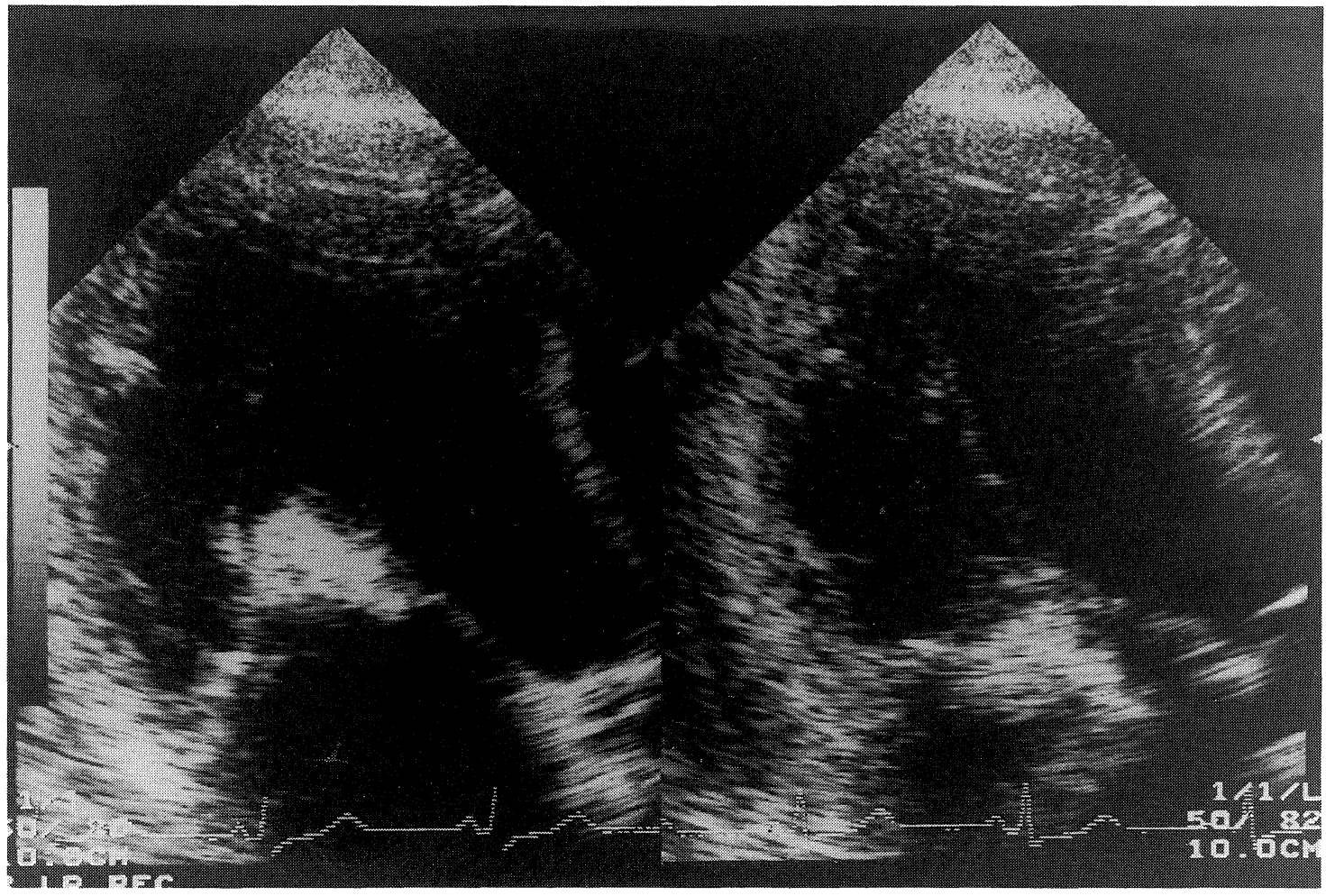

Figure 2. A transesophageal echocardiograph reveals invagination into the mitral valve during diastole. 

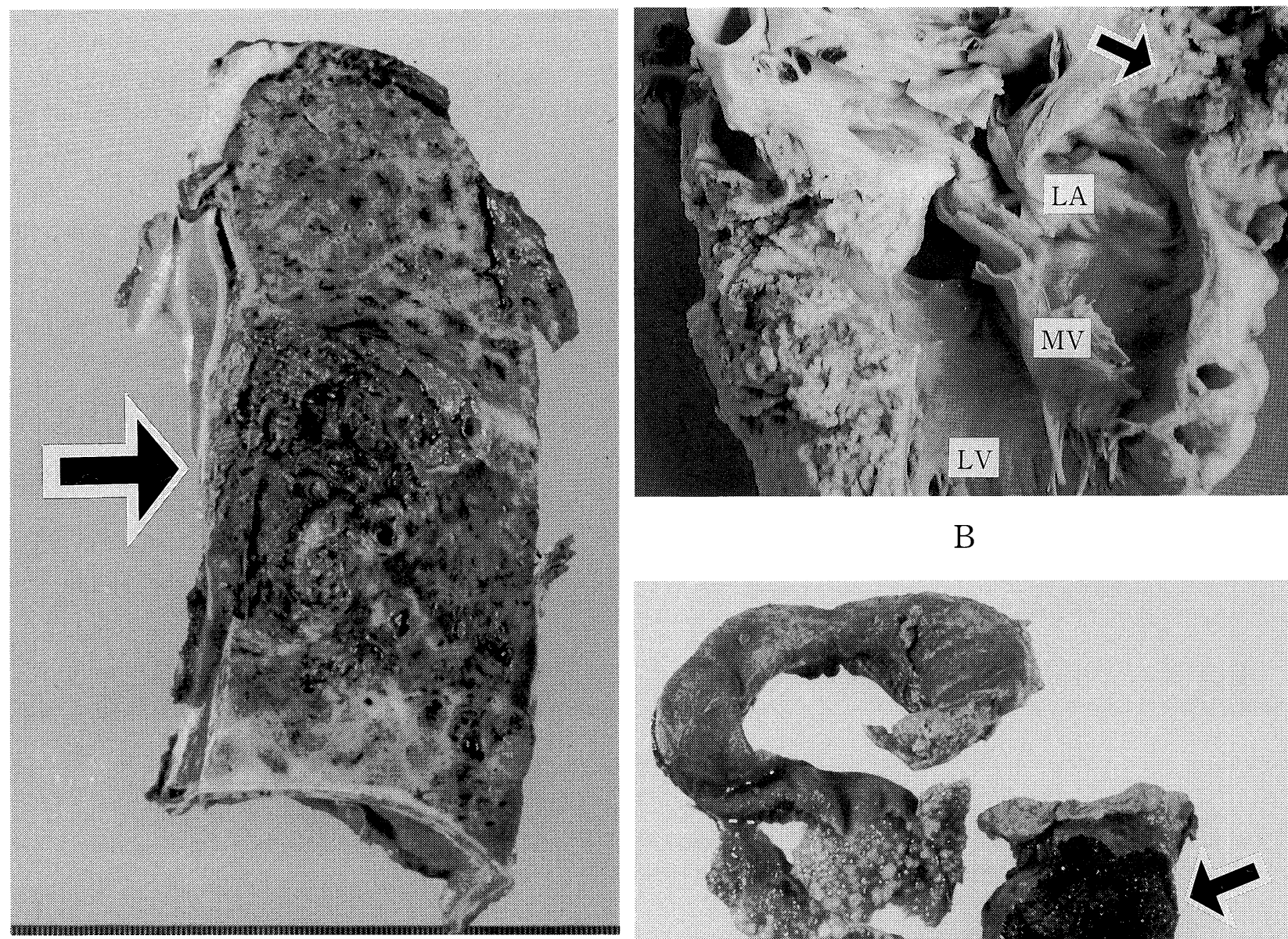

B

A

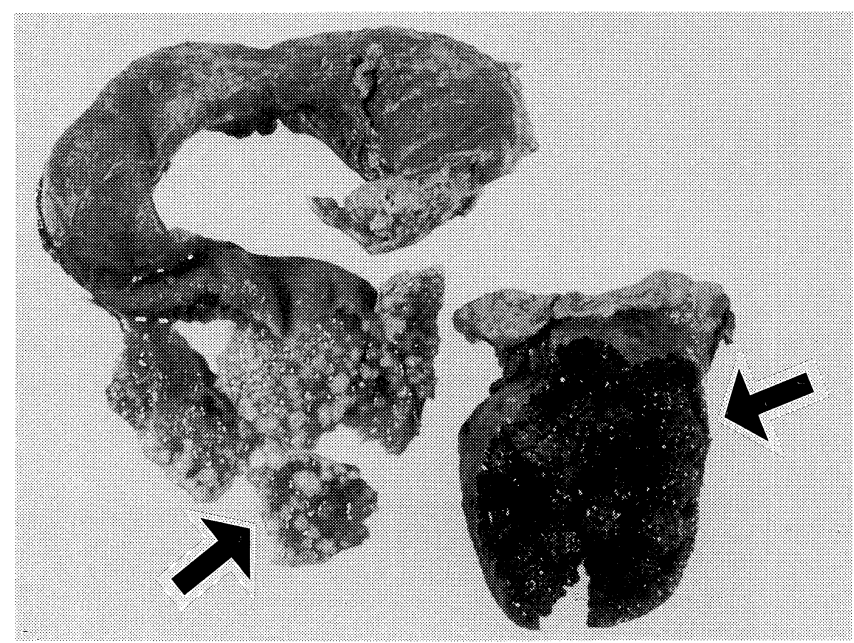

$\mathrm{C}$

Figure 3. A) Macroscopic examination reveals necrotic mass in the left lung (arrow). B) Cut surface of the heart shows the invaded pulmonary vein (arrow). C) The mass with stalk is protruded into the LA: the small arrow shows the site of connection to the LA endocardium, and the big arrow shows the protruded mass in the LA.

The mass extended into the left lower pulmonary vein (Fig. 3B), and protruded as a polyp with stalk into the LA (Fig. 3C). The histopathologic examination showed the polypoid lesion in the LA was composed of thrombus (Fig. 4A), and hyphae of aspergillus were found in the left lung mass and left atrial wall (Fig. 4B). Direct invasion of pulmonary aspergillosis into the left atrial wall was observed.

\section{Discussion}

Pulmonary aspergillosis is classified in three forms: invasive broncho-pulmonary aspergillosis (pneumonia type), aspergilloma and allergic aspergillosis. Binder et al proposed the concept that chronic necrotizing pulmonary aspergillosis is the chronic form of invasive pulmonary aspergillosis (1). Which of these forms occur maybe dependent on the reciprocal action of the host immune system and the pathogenicity of aspergillus species (1-3). In this case, the time from hospital visit to death was one year. The clinical course took long time, and her disease state progressively deteriorated and the fungicidal treatment was not effective. She had been treated for diabetes mellitus, but the control of diabetes was poor. We considered this case as chronic necrotizing pulmonary aspergillosis. On admission, we suspected that the left lung mass shadow was lung cancer with direct cardiac invasion, however, the lung biopsy specimens showed no evidence of malignancy, and hyphae of Aspergillus fumigatus were found in the specimens. Therefore, we suspected that the cardiac lesion resulted from 


\section{Pulmonary Aspergillosis with Cardiac Invasion}

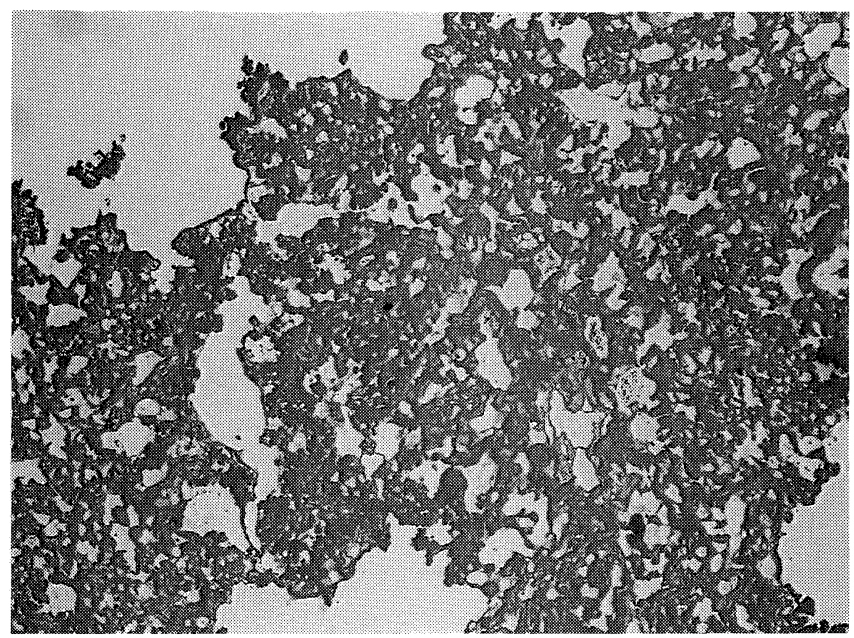

A

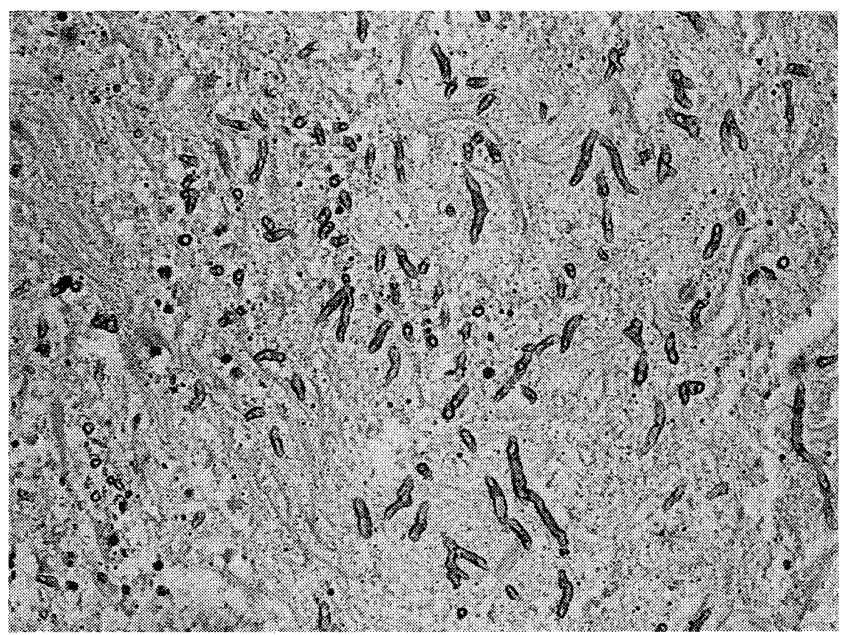

B

Figure 4. A) The LA mass is composed of thrombus (HE stain, $\times 400)$. B) Fungal hyphae are seen in the LA wall (PAS stain, $\times 400)$.

pulmonary aspergillosis or a combination of pulmonary aspergillosis and a cardiac tumor such as LA myxoma. Although she also developed left hemiparesis while in hospital, brain CT scans revealed no significant abnormalities, and her hemiparesis improved gradually, suggesting cerebral embolism from the LA mass. Postmortem examination confirmed the presence of pulmonary aspergillosis, which had extended into the left atrial wall via pulmonary vein, and thrombus with stalk was formed in the LA. We considered that endocardium of the LA was invaded by aspergillus from the lung and thrombus was formed on the injured site. Finally, we established the diagnosis as direct LA invasion of pulmonary aspergillosis with thrombus. There have been some case reports of invasive aspergillosis with myocardial invasion. In those case reports, the LA mass revealed hyphae of Aspergillus species (4). In our case, the LA mass was composed of thrombus, and fungal hyphae were observed in the LA endocardium. We should have treated not only the infection of Aspergillus but also thrombus. Invasive aspergillosis in an immunocompromised host has been documented $(5,6)$. However, there have been few reports of invasive aspergillosis in immunocompetent hosts $(7,8)$.

The present patient had been treated for diabetes mellitus. In patients with diabetes mellitus, granulocytic chemotaxis does seem to be impaired independently of ketoacidosis, although it can be restored with antioxidants (9). Although we do not know whether or not the diabetes mellitus affected the onset of fungal infection in this case, the patient had no other immunologic abnormalities. Cardiac infection by aspergillus is rare and is usually diagnosed by postmortem examination $(10,11)$. In most cases with cardiac invasion, the terminal event may be embolization of a large portion of the atrial mass. We thought that our patient suffered from the result of invagination of polypoid thrombus in the mitral valve and died of mitral obstruc- tion. The prognosis of aspergillosis with cardiac invasion is very poor, usually due to the late diagnosis and the presence of a serious associated disease (12-18). In the present case, although the fungicidal treatment was not effective; she had a large tumor in the LA, and her general condition was poor, therefore we could not choose an operation. If more early diagnosis was performed, we might have choosen operation of the left lung and left atrial resection. A delayed diagnosis may result in poor prognosis. Heightened clinical suspicion and early cardiac imaging are necessary for prompt diagnosis. Earlier diagnosis and treatment could improve the outcome of this usually fatal disease.

\section{References}

1) Binder RE, Faling LJ, Pugatch RD, et al. Chronic necrotizing pulmonary aspergillosis. Medicine (Baltimore) 61: 109-124, 1982.

2) Mizuki M, Chikuba K, Tanaka K. A case of chronic necrotizing pulmonary aspergillosis due to Aspergillus nidulans. Mycopathologia 128: 7579, 1994.

3) Nagai H. Saprophytic and invasive pulmonary aspergillosis. Kekkaku 72: 99-107, 1997 (in Japanese).

4) Berarducci L, Ford $K$, Olenick $S$, et al. Invasive intracardiac aspergillosis with widespread embolization. J Am Soc Echocardiogr 6: 539-542, 1993.

5) Karim M, Alam M, Shah AA, et al. Chronic invasive aspergillosis in apparently immunocompetent hosts. Clin Infect Dis 24: 723-733, 1997.

6) Tritz DM, Woods GL. Fatal disseminated infection with Aspergillus terreus in immunocompromised hosts. Clin Infect Dis 16: 118-122, 1993.

7) Cooper JA, Weinbaum DL, Aldrich TK, et al. Invasive aspergillosis of the lung and pericardium in a nonimmunocompromised 33 year old man. Am J Med 71: 903-907, 1981.

8) Rosenberg RS, Creviston SA, Schonfeld AJ. Invasive aspergillosis complicating resection of a pulmonary aspergilloma in a nonimmunocompromised host. Am Rev Respir Dis 126: 1113-1115, 1982.

9) Fishman AP, Fishman JA, Grippi MA, et al. Fishman's Pulmonary Disease and Disorders, 3rd ed. New York, McGraw-Hill, 1998: 2082.

10) Walsh TJ, Hutchins GM, Bulkley BH, et al. Fungal infections of the heart: 


\section{KoBAYASHI et al}

Analysis of 51 autopsy cases. Am J Cardiol 45: 357-366, 1980.

11) Atkinson JB, Connor DH, Robinowitz $M$, et al. Cardiac fungal infections. Review of autopsy findings in 60 patients. Human Pathol 15: 935942, 1984.

12) Mullen P, Jude C, Borkon M, et al. Aspergillus mural endocarditis. Clinical and echocardiographic diagnosis. Chest 90: 451-452, 1986.

13) Andersson BS, Luna MA, McCredie KB. Systemic aspergillosis as cause of myocardial infarction. Cancer 58: 2146-2150, 1986.

14) Rinaldi MG. Invasive aspergillosis. Rev Infect Dis 5: 1061-1077, 1983.

15) Robbins JA, Mohanty PK, Mohanty LB, et al. Aortic aspergilloma result- ing in supravalvular aortic stenosis after aortocoronary bypass surgery. Am Heart J 106: 163-166, 1983.

16) Rogers JG, Windle JR, McManus BM, et al. Aspergillus myocarditis presenting as myocardial infarction with complete heart block. Am Heart $\mathrm{J}$ 120: 430-432, 1990.

17) Andi NM, Condos WR Jr, Tami LF, et al. Images in cardiovascular medicine. Aspergillus mitral endocarditis. Circulation 96: 4431, 1997.

18) Woods GL, Wood RP, Shaw BW Jr. Aspergillus endocarditis in patients without prior cardiovascular surgery. Report of a case in a liver transplant recipient and review. Rev Infect Dis 11: 263-272, 1989. 